\title{
Validation and factor analysis of mother- infant bonding questionnaire in pregnant and postpartum women in Japan
}

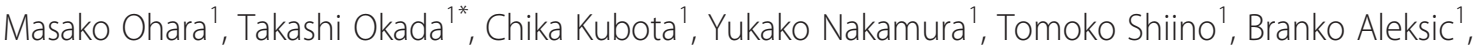 \\ Mako Morikawa', Aya Yamauchi ${ }^{1}$, Yota Uno ${ }^{1}$, Satomi Murase', Setsuko Goto², Atsuko Kanai ${ }^{3}$, Tomoko Masuda ${ }^{4}$ \\ and Norio Ozaki ${ }^{1}$
}

\begin{abstract}
Background: The Mother-Infant Bonding Questionnaire (MIBQ) has been widely used to assess maternal emotional involvement with infants. Although the reliability and validity of the MIBQ in the postpartum period has been confirmed, it remains unclear whether the MIBQ is appropriate to assess maternal bonding in both pregnancy and the postpartum period over time. Our study were aimed to 1) examine the reliability and validity of the MIBQ for clinical use among pregnant and postpartum women; and 2) examine the factor structure of the items, create subscales, and confirm the stability of the MIBQ in the pregnancy and postpartum periods.
\end{abstract}

Methods: Participants ( $n=751$, mean age $32.1 \pm 4.4$ years) completed the MIBQ and the Edinburgh Postnatal Depression Scale (EPDS) in early pregnancy (before week 25), in late pregnancy (around week 36), 5 days after delivery, and 1 month after delivery. We randomly divided participants into two sample sets. We conducted an exploratory factor analysis of the nine MIBQ items using data from one group of mothers (Group $1 ; n=376$ ) in all four periods. The factor structure derived from the exploratory factor analysis was confirmed by a confirmatory factor analysis in the second group (Group 2; $n=375$ ) of mothers in all four periods.

Results: Exploratory factor analysis yielded two factors: Lack of Affection (LA) and Anger and Rejection (AR). Confirmatory factor analysis demonstrated that LA and AR factors existed for the MIBQ in all periods. Cronbach's alpha coefficients were 0.879 and 0.584 , respectively. The scores for LA and AR were significantly correlated over the four time periods. Mothers with higher AR scores on the MIBQ at any of the four periods had higher scores on the EPDS.

Conclusions: The MIBQ has two subscales regardless of the timing of the assessment. The MIBQ is appropriate for pregnant as well as postpartum women to assess maternal bonding toward the fetus and infant.

\section{Background}

Maternal emotional feelings and loving attitude toward their infant lead to establishment of an effective attachment system. This important psychological process was named "bonding" [1]. Kumar reported that some mothers showed a delay in the development of maternal bonding, resulting in infant neglect or impulses to harm the infant [2]. This finding was supported by

\footnotetext{
* Correspondence: okada@med.nagoya-u.ac.jp

1 Department of Psychiatry, Nagoya University Graduate School of Medicine, 65 Tsurumai-cho, Showa-ku, Nagoya, Aichi 466-8550, Japan

Full list of author information is available at the end of the article
}

subsequent observational studies. For example, crosssectional studies suggested that poor mother-infant bonding was associated with less interactive behaviors $[3,4]$. Kitamura et al. performed path analysis to elucidate causal relationships between maternal bonding and abusive parenting, and indicated that bonding failure in the postpartum period predicted abusive parenting [5]. In addition, a longitudinal study also showed that bonding impairment in the postpartum period was related to insecure parenting behaviors [6].

Maternal bonding starts to develop before a child is born [7]. Observational studies examined whether 
negative maternal involvement with the fetus was correlated with adverse effects on the infant's outcome. Alhusen et al. reported that the children whose mothers reported lower maternal emotional involvement during pregnancy had less optimal early childhood development and less secure interactions with their mothers [8].

In addition, it has been suggested that maternal bonding in pregnancy predicts maternal bonding in the postpartum period. In a longitudinal study, women with more feelings of closeness and tenderness toward their fetuses in pregnancy reported more feelings of pleasure and affiliation in the relationship with their infants $[9,10]$. These results suggest that maternal bonding disturbances start in pregnancy and are related to impairments in the mother-child relationship, which may result in maltreatment or child abuse. To ensure a positive relationship between the mother and infant in the postpartum period, it is essential to detect maternal bonding disturbances earlier in the perinatal period, and to follow up on the status of these disturbances. Therefore, it is important to assess maternal bonding during pregnancy and the postpartum period over time, using the same instruments. However, there have been no appropriate instruments to assess maternal bonding in both pregnancy and the postpartum period over time. The Postpartum Bonding Questionnaire (PBQ) has been standardized for the postpartum period [11]. Some items on the PBQ are applicable only to postpartum women (e.g., "I feel happy when my baby smiles or laughs). The Maternal Antenatal Attachment Scale (MAAS) is a widely used measure of maternal-fetal attachment during pregnancy [12]. Some items on the MAAS are applicable only to pregnant women (e.g., "Frequent/infrequent picturing of the fetus in my imagination").

Kumar developed a self-report screening scale, based on the mother's narrative accounts [2]. This scale was named the Mother-Infant Bonding Questionnaire (MIBQ), which has nine items: "loving", "disappointed", "neutral or felt nothing", "possessive", "resentful", "dislike", "protective", "joyful", and "aggressive" [13, 14]. All items on the MIBQ are applicable during for the pregnancy and postpartum periods. The MIBQ does not require a high reading level, and takes only a few minutes to complete. Although the reliability and validity of the MIBQ in the postpartum period has been confirmed [13], it remains unclear whether the MIBQ is also appropriate during pregnancy.

Therefore, the aims of our study were as follows: 1) to examine the reliability and validity of the MIBQ for clinical use among pregnant and postpartum women; and 2) to examine the factor structure of the items, create subscales, and confirm the stability of the MIBQ in the pregnancy and postpartum periods.

\section{Methods}

\section{Participants}

Participants in this study were recruited from perinatal classes for pregnant women (starting before week 25 of pregnancy) at two obstetrical hospitals and one university hospital in central Nagoya, Japan (with a population of approximately 2 million) between August 2004 and March 2015. Mothers with current or past histories of mental illness were excluded from the study, as well as mothers with children born before week 32 of gestation. In addition, participants were required to be at least 20 years old and capable of understanding the Japanese language.

\section{Procedures}

Pregnant women attending perinatal classes were given detailed information about the study design and methods. This information was given orally and on paper in the three hospitals. Women who agreed to cooperate in the study were asked to complete self-reporting questionnaires, which included social demographic questions, the MIBQ, and the Edinburgh Postnatal Depression Scale (EPDS) in early pregnancy before week 25 (T1), and return them by mail. After receiving the completed consent forms and questionnaires, the MIBQ and the EPDS questionnaires were sent again around week 36 of pregnancy (T2) and returned by mail. At day 5 and 1 month after delivery (T3, T4), the MIBQ and the EPDS were sent and returned by mail. A total of 990 women agreed to participate at the perinatal classes starting before week 25 of pregnancy; 751 (75.9 \%) mothers completed all questionnaires for all periods.

\section{Measures \\ Mother-Infant Bonding Questionnaire (MIBQ)}

The MIBQ is a self-reporting scale designed to assess maternal bonding with their baby during the postpartum period; it is composed of nine items: "loving", "disappointed", "neutral or felt nothing", "possessive", "resentful", "dislike", "protective", "joyful", and "aggressive" [13, 14]. The MIBQ is rated on a four-point Likert scale (from 0 , "very much" to 3 , "not at all"), with the scale of some items reversed. Total scores range from 0 to 27. A high score indicates worse mother to infant bonding. The reliability and validity of the MIBQ in the postpartum period have been reported [13]. Yamashita translated the MIBQ into Japanese, and then the Japanese version was retranslated back into English by a native English translator who was unaware of the original wording to confirm that the translation was consistent with the original meaning. This version was used in a previous clinical study in Japan [15]. The reliability and validity of the Japanese version of the MIBQ have not been reported. 


\section{Edinburgh Postnatal Depression Scale (EPDS)}

The EPDS is a self-reporting questionnaire designed to assess postpartum depression; it is composed of 10 items scored on a four-point Likert scale [16]. Numerous studies have used this instrument during pregnancy and the postpartum period. The EPDS Japanese version showed good internal consistency (Cronbach's alpha $=0.78$ ) and testretest reliability (Spearman's correlation $=0.92$ ) [17]. A score $\geq 9$ was designated to screen for minor and major depressive episodes, with a sensitivity of $75 \%$ and $82 \%$ and a specificity of $93 \%$ and $95 \%$, respectively $[17,18]$.

\section{Statistical analysis}

First, participants ( $n=751$, mean age $32.1 \pm 4.4$ years) were randomly divided into two groups (Group 1, $n=376$; Group 2, $n=375$ ) using a random sampling technique. We calculated descriptive statistics for the nine items of the MIBQ as shown in Table 1. Most of the items were positively skewed (skew values greater than 1) at all time points. We therefore log-transformed all MIBQ scores for the subsequent factor analysis. We conducted an exploratory factor analysis (EFA) of nine items of the MIBQ using data from Group 1. Because all factors were considered dependent upon each other, the factor solution was sought after Promax rotation, which is an oblique rotation. The number of factors was determined by scree plot [19]. To create a subscale of the MIBQ, we extracted items for each subscale if they were loaded $\geq 0.3$ on a particular factor but $<0.3$ on the other factors.

Second, the factor structure derived from the EFA was confirmed by a confirmatory factor analysis (CFA) in Group 2. The fit of each model with the data was examined in terms of chi-squared (CMIN), degree of freedom (df), comparative fit index (CFI), and root mean square error of approximation (RMSEA).
According to conventional criteria, CMIN $/ d f<2$, CFI $>$ 0.97 , and RMSEA $<0.05$ indicate a good fit, while CMIN/ $d f<3$, CFI $>0.95$, and RMSEA $<0.08$ indicate an acceptable fit [20]. The Akaike Information Criterion (AIC) was used to compare different models; a model with an AIC score at least two points lower is regarded as a better model.

Cronbach's alpha for the two hypothesized subscales was calculated to examine the internal reliability of the MIBQ.

Previous studies reported that maternal depressive mood associated with bonding disturbances [21-23]. In addition, in previous studies, EPDS was used as a way to assess construct validity for other instruments to assess bonding disturbances [14]. In accordance with previous research, we constructed a correlation matrix with the EPDS. This analysis provided evidence of construct validity.

All statistical analyses were conducted using the SPSS version 22.0 and Amos 21.0 (IBM Japan, Tokyo, Japan).

\section{Results}

\section{Descriptive statistics}

The means and SDs of all MIBQ items in early pregnancy before week 25 (T1), late pregnancy around week 36 (T2), 5 days after delivery (T3), and 1 month after delivery (T4) were relatively low (Table 1 ). In Table 1 , total scores at T3 $(1.54 \pm 2.30)$ were lower than other points; $\mathrm{T} 1$ (3.11 \pm 3.03$)$, T2 $(2.95 \pm 2.93)$, and T4 $(1.78 \pm 2.45)$. Total scores decreased from $\mathrm{T} 1$ to $\mathrm{T} 2$. Total scores increased from T3 to T4. This is in line with previous report [24]. Most of the items were positively skewed. We therefore log-transformed all scores for MIBQ items before entering them into the EFA.

\section{Factor analysis of MIBQ items}

All the log-transformed items of the MIBQ were entered into an EFA. This suggested a two-factor structure (Table 2). The first factor was loaded by five items:

Table 1 Means and SDs of the MIBQ items at the four time points in Group 1

\begin{tabular}{|c|c|c|c|c|c|c|c|c|}
\hline \multirow[t]{2}{*}{ MIBQ items } & \multicolumn{2}{|l|}{$\mathrm{T} 1$} & \multicolumn{2}{|l|}{$\mathrm{T} 2$} & \multicolumn{2}{|l|}{ T3 } & \multicolumn{2}{|l|}{ T4 } \\
\hline & M (SD) & Skewness & $M(S D)$ & Skewness & $M(S D)$ & Skewness & M (SD) & Skewness \\
\hline 1: loving & $0.45(0.65)$ & 1.28 & $0.39(0.59)$ & 1.29 & $0.10(0.35)$ & 3.99 & $0.09(0.30)$ & 3.58 \\
\hline 2: disappointed & $0.06(0.30)$ & 5.87 & $0.05(0.31)$ & 6.45 & $0.07(0.35)$ & 5.61 & $0.09(0.40)$ & 5.47 \\
\hline 3: neutral and felt nothing & $0.42(0.80)$ & 1.75 & $0.44(0.83)$ & 1.82 & $0.26(0.72)$ & 2.89 & $0.19(0.61)$ & 3.36 \\
\hline 4: possessive & 1.09 (1.03) & 0.45 & $0.90(0.96)$ & 0.69 & $0.59(0.90)$ & 1.32 & $0.62(0.88)$ & 1.26 \\
\hline 5: resentful & $0.04(0.25)$ & 7.08 & $0.04(0.24)$ & 6.18 & $0.07(0.32)$ & 5.07 & $0.24(0.55)$ & 2.22 \\
\hline 6: dislike & $0.10(0.41)$ & 4.71 & $0.11(0.48)$ & 4.70 & $0.07(0.36)$ & 5.68 & $0.13(0.45)$ & 3.79 \\
\hline 7: protective & $0.30(0.60)$ & 2.04 & $0.34(0.61)$ & 1.77 & $0.13(0.42)$ & 3.64 & $0.09(0.32)$ & 3.96 \\
\hline 8: joyful & $0.61(0.82)$ & 1.16 & $0.64(0.81)$ & 1.01 & $0.22(0.54)$ & 2.73 & $0.25(0.52)$ & 2.01 \\
\hline 9: aggressive & $0.06(0.32)$ & 5.57 & $0.04(0.25)$ & 8.10 & $0.03(0.22)$ & 9.49 & $0.09(0.34)$ & 4.29 \\
\hline Total & $3.11(3.03)$ & 1.24 & $2.95(2.93)$ & 1.19 & $1.54(2.30)$ & 3.03 & $1.78(2.45)$ & 2.17 \\
\hline
\end{tabular}

Each item is scored on a four-point Likert scale ranging from 0 to 3 . Total scores can range from 0 to 27 MIBQ Mother-Infant Bonding Questionnaire, $M$ mean, SD standard deviation, $T 1$ early pregnancy before week $25, T 2$ late pregnancy around week $36, T 35$ days after delivery, T4 1 month after delivery 
Table 2 Factor structure of the MIBQ items at early pregnancy before week 25 in Group 1

\begin{tabular}{lll}
\hline MIBQ items & Factor 1 & Factor 2 \\
\hline 8: joyful & $\mathbf{. 8 1 9}$ & -.005 \\
1: loving & $\mathbf{. 8 1 0}$ & -.030 \\
7: protective & $\mathbf{. 7 1 8}$ & .104 \\
4: possessive & $\mathbf{. 5 1 7}$ & -.167 \\
3: neutral or felt nothing & $\mathbf{. 3 0 0}$ & .180 \\
6: dislike & -.068 & $\mathbf{. 6 8 8}$ \\
5: resentful & -.010 & $\mathbf{. 6 8 2}$ \\
2: disappointed & .181 & .299 \\
9: aggressive & -.038 & .253 \\
\hline
\end{tabular}

Factor loadings $\geq 0.3$ are bold

MIBQ Mother-Infant Bonding Questionnaire

"loving", "neutral or felt nothing", "possessive", "protective", and "joyful". These items reflected lack of positive affection and intimacy toward the baby. We named this factor Lack of Affection (LA). The second factor was loaded by two items: "resentful" and "dislike". These items reflected a mother's anger and rejection toward the baby. We named this factor Anger and Rejection (AR).

The factor structure extracted in the EFA of the MIBQ was then subjected to a CFA using Group 2. The model derived from the EFA showed good fit with the data $(\mathrm{CMIN} / d f=1.264, \mathrm{CFI}=0.995, \mathrm{RMSEA}=0.027)($ Fig. 1$)$.

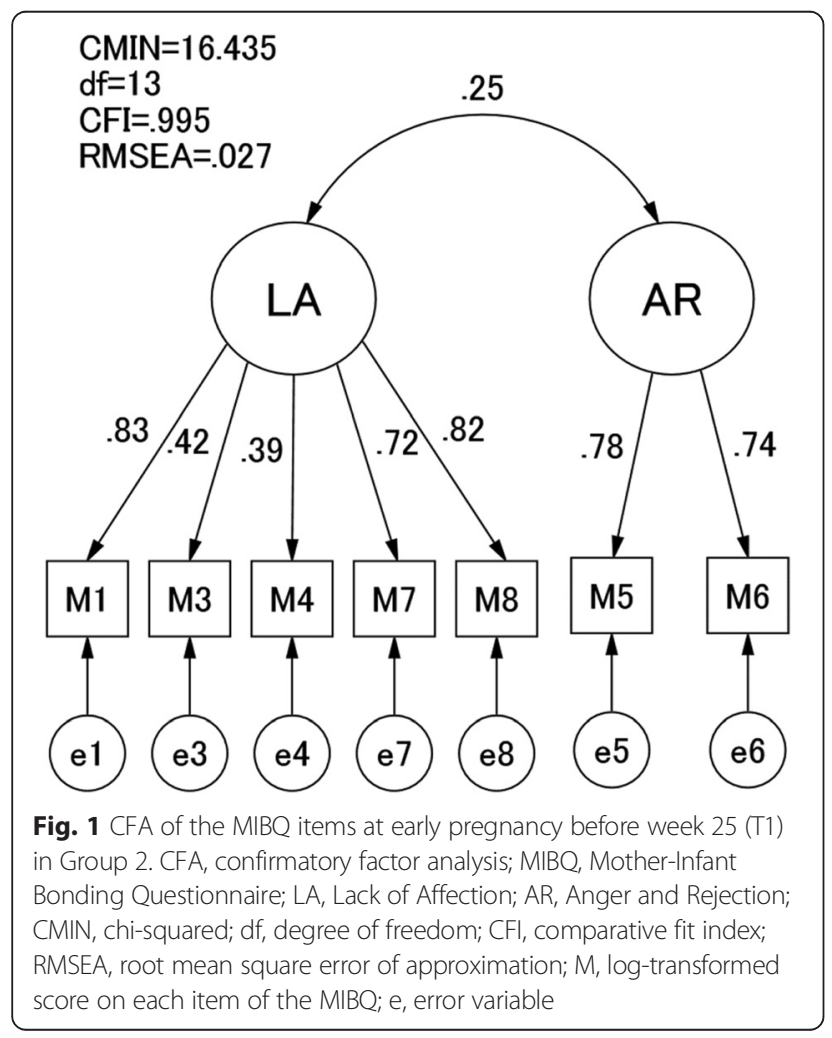

The two latent factors were moderately correlated with each other.

To examine stability of the factor structure of the MIBQ, we conducted a series of CFAs using all the periods from Group 2. The path values and accompanying fit indices are shown in Table 3. The correlation between the LA and AR factors was moderate in all four periods. For all four periods, CMIN/df values were $<2$, indicating a good model of fit [20]. Examination of CFI and RMSEA revealed the current model to meet good fit criteria for all four periods. The fitness of the model to data was good for data from all four periods.

\section{Internal consistency of mother-infant bonding questionnaire}

As for the combined data of Group 1, internal consistency (Cronbach's alpha) of the five items belonging to LA was 0.879 , whereas that of two items belonging to $\mathrm{AR}$ was 0.584 , showing reasonable internal consistency.

\section{Construct validity of mother-infant bonding questionnaire} Both LA and AR subscales were positively correlated with the EPDS scores in all four periods (Table 4). Thus, negative affect of mothers correlated with worse bonding. The strongest correlations were between AR and EPDS, especially at 1 month postpartum.

Table 3 CFA of the MIBQ items over the four time points in Group 2

\begin{tabular}{lllll}
\hline MIBQ items & T1 & T2 & T3 & T4 \\
\hline Lack of Affection (LA) & & & & \\
1: Loving & 0.83 & 0.85 & 0.71 & 0.65 \\
3: Neutral or felt nothing & 0.42 & 0.36 & 0.18 & 0.14 \\
4: Possessive & 0.39 & 0.45 & 0.37 & 0.37 \\
7: Protective & 0.72 & 0.72 & 0.7 & 0.52 \\
8: Joyful & 0.82 & 0.75 & 0.68 & 0.76 \\
Anger and Rejection (AR) & & & & \\
5: Resentful & 0.78 & 0.75 & 0.3 & 0.57 \\
6: Dislike & 0.74 & 0.59 & 0.16 & 0.69 \\
Covariance between LA and AR & 0.25 & 0.34 & 0.23 & 0.29 \\
CMIN/df & 1.264 & 1.423 & 0.94 & 1.09 \\
CFI & 0.995 & 0.991 & 1 & 0.996 \\
RMSEA & 0.027 & 0.034 & 0 & 0.016 \\
AIC & 46.435 & 48.499 & 42.215 & 44.175 \\
\hline
\end{tabular}

CFA confirmatory factor analysis, MIBQ Mother-Infant Bonding Questionnaire, $T 1$ early pregnancy before week 25, T2 late pregnancy around week $36, T 3$ 5 days after delivery, T4 1 month after delivery, LA lack of affection, $A R$ Anger and Rejection, CMIN/df chi-squared/degree of freedom, CFI comparative fit index, RMSEA root mean square error of approximation 
Table 4 Pearson's correlations of MIBQ subscale scores and EPDS, $n=751$

\begin{tabular}{|c|c|c|c|c|c|c|c|c|c|c|c|c|c|}
\hline & & \multicolumn{2}{|l|}{$\mathrm{T1}$} & \multicolumn{2}{|l|}{ T2 } & \multicolumn{2}{|l|}{ T3 } & \multicolumn{2}{|l|}{ T4 } & \multirow{2}{*}{$\begin{array}{l}\text { EPDS } \\
\mathrm{T} 1\end{array}$} & \multirow{2}{*}{$\begin{array}{l}\text { EPDS } \\
\text { T2 }\end{array}$} & \multirow{2}{*}{$\begin{array}{l}\text { EPDS } \\
\text { T3 }\end{array}$} & \multirow{2}{*}{$\begin{array}{l}\text { EPDS } \\
\text { T4 }\end{array}$} \\
\hline & & $\overline{\mathrm{LA}}$ & AR & $\overline{\mathrm{LA}}$ & AR & $\overline{L A}$ & $A R$ & $\overline{\mathrm{LA}}$ & $A R$ & & & & \\
\hline \multirow[t]{2}{*}{$\mathrm{T} 1$} & LA & & $0.21^{* *}$ & $0.73^{* *}$ & $0.20^{* *}$ & $0.43^{* *}$ & 0.06 & $0.48^{* *}$ & $0.24^{* *}$ & $0.14^{* *}$ & & & \\
\hline & $A R$ & & & $0.20^{* *}$ & $0.48^{* *}$ & $0.07^{*}$ & $0.27^{* *}$ & 0.07 & $0.28^{* *}$ & $0.25^{* *}$ & & & \\
\hline \multirow[t]{2}{*}{$\mathrm{T} 2$} & LA & & & & $0.21^{* *}$ & $0.46^{* *}$ & 0.06 & $0.50^{* *}$ & $0.22^{* *}$ & & $0.18^{* *}$ & & \\
\hline & $A R$ & & & & & $0.14^{* *}$ & $0.36^{* *}$ & $0.18^{* *}$ & $0.43^{* *}$ & & $0.25^{* *}$ & & \\
\hline \multirow[t]{2}{*}{ T3 } & LA & & & & & & $0.18^{* *}$ & $0.66^{* *}$ & $0.24^{* *}$ & & & $0.17^{* *}$ & \\
\hline & $A R$ & & & & & & & $0.14^{* *}$ & $0.38^{* *}$ & & & $0.25^{* *}$ & \\
\hline \multirow[t]{2}{*}{$\mathrm{T} 4$} & LA & & & & & & & & $0.26^{* *}$ & & & & $0.23^{* *}$ \\
\hline & $A R$ & & & & & & & & & & & & $0.33^{* *}$ \\
\hline
\end{tabular}

MIBQ, Mother-Infant Bonding Questionnaire, EPDS Edinburgh Postnatal Depression Scale, $T 1$ early pregnancy before week $25, T 2$ late pregnancy around week 36, T3 5 days after delivery, T4 1 month after delivery, LA lack of affection, AR Anger and Rejection

${ }^{*} p<0.05$

${ }^{* *} p<0.01$

\section{Test-retest reliability of mother-infant bonding questionnaire}

Pearson's correlations of MIBQ subscales are shown in Table 4. The scores of LA were significantly correlated over the four time points (all $p<0.01$ ). Those of AR were also significantly correlated over the four time points (all $p<0.01$ ).

\section{Discussion}

To the best of our knowledge, the present study is the first to validate the MIBQ, examine the factor structure of the items, create subscales, and confirm stability in both the pregnancy and postpartum periods.

The reliability and validity of the MIBQ were examined among subjects during pregnancy and in the postpartum period. In this cohort, the two-factor structure, LA and AR, was confirmed in all four periods. The internal consistencies of the two factors were reasonable. The two subscales of the MIBQ were positively correlated with EPDS scores over time and demonstrated acceptable construct validity. In addition, the scores of LA were significantly correlated over the four time points, as were the scores of AR. These results demonstrated the stability of the MIBQ over the periods of the current study. Therefore, the MIBQ should be considered a reasonable scale to assess maternal emotional involvement with the fetus and infant during pregnancy and in the postpartum period.

The strength of our study is that we conducted an EFA for half of the participants (Group 1;n=376) and confirmed stability of factor structure in the other half (Group 2; $n=375$ ) to compare our four time points. The present study suggested that the MIBQ items fit the two-factor model for data in EFA. Taylor et al. also investigated the psychometric properties of the MIBQ [13]. He examined inter-item correlations of the MIBQ by using principal components analysis, and proposed that the original nine-item Kumar's MIBQ score fit a onefactor model after discarding one item (item 4; possessive). We also tested Taylor's one-factor model. The results did not show goodness-of-fit with our data $(\mathrm{CMIN} / d f=13.42$, $\mathrm{CFI}=0.691, \mathrm{RMSEA}=0.182, \mathrm{AIC}=300.328)$. Therefore, our two-factor model had an adequate fit to our data and was superior in statistical significance to the one-factor model.

According to our two-factor model, the MIBQ is likely to detect LA and AR. These subscales correspond with the factors that were proposed in previous studies using other instruments that assessed maternal bonding (PBQ [11], Mother-to-Infant Bonding Scale [5, 14]). Our two subscales correspond with "Impaired bonding" and "Rejection and Anger" in the 25-item PBQ by Brockington et al. that revealed a four-factor structure. Brockington et al. examined PBQ and proposed the following four-factor model: Scale 1 is "Impaired bonding"; Scale 2 is "Rejection and Anger"; Scale 3 may be useful in anxious mothers; and Scale 4 signaled the presence of incipient abuse [11]. In addition, our two subscale correspond with "Lack of Affection" and "Anger and Rejection" in the 10-item Japanese version of the Mother-to-Infant Bonding Scale by Yoshida et al. and Kitamura et al. that revealed a twofactor structure $[5,14]$.

It is should be emphasized that the EPDS showed correlations with both LA and AR. This means that both LA and AR were associated with the psychopathology of depressive symptoms. Furthermore, the correlation was especially large in the AR factor over the four periods. This finding suggests that depressive symptoms might be especially linked with anger and rejection toward a child. This study elucidated the associations between depressive symptoms and bonding disturbances. This is in line with previous reports. For example, Moehler et al. examined the relationship between postnatal depression and maternal bonding to the infant with the PBQ and 
EPDS using multivariate analysis. They reported that maternal depressive symptoms in the postnatal period were associated with long-term impairment of motherchild bonding [25]. In addition, Sockol also investigated correlates of mother-infant bonding among postpartum women with the PBQ and EPDS using multiple regression analysis. They reported that one of the strongest predictors of impairments in mother-infant bonding was maternal depressive symptom level [26]. However, there are numerous variables known to be associated with bonding disturbances and depressive symptoms. Further study is needed to elucidate these two issues.

Several limitations of this study need to be addressed. First, participants of this study were recruited from perinatal classes for pregnant women, and they participated in this study voluntarily. Therefore, this sample may not be representative of the total population. Second, this study was based only on self-administered questionnaires without diagnostic procedures based on external criteria. Therefore, criterion validity was not investigated in this study.

\section{Conclusions}

The results of the present study indicate that the MIBQ should be considered a reasonable scale to assess maternal emotional involvement with the fetus and infant during pregnancy and in the postpartum period. In addition, the MIBQ has two subscales, LA and AR. The EPDS showed a correlation with both LA and AR. This study suggests associations between depressive symptoms and bonding disorder. These results indicate that the MIBQ is appropriate for pregnant as well as postpartum women to assess maternal bonding toward a fetus and infant.

\section{Abbreviations \\ AIC, Akaike Information Criterion; AR, anger and rejection; CFA, confirmatory factor analysis; $C F$, comparative fit index; CMIN, chi-squared; DF, degree of freedom; EFA, exploratory factor analysis; EPDS, Edinburgh Postnatal Depression Scale; LA, lack of affection; MAAS, Maternal Antenatal Attachment Scale; MIBQ, Mother-Infant Bonding Questionnaire; PBQ, Postpartum Bonding Questionnaire; RMSEA root mean square error of approximation; $T 1$, early pregnancy before week $25 ; T 2$, late pregnancy around week $36 ; T 3,5$ days after delivery; $T 4,1$ month after delivery}

\section{Acknowledgements}

We would like to express our gratitude to the staff of Nagoya University Hospital, Nagoya Teishin Hospital, and Kaseki Hospital for their cooperation. We are grateful to Dr. T. Kitamura for technical advice.

\section{Funding}

Funding for this study was provided by research grants from the Ministry of Education, Culture, Sports, Science and Technology of Japan; the Ministry of Health, Labor and Welfare of Japan; Intramural Research Grant (21B-2) for Neurological and Psychiatric Disorders of National Center for Neurology and Psychiatry. The funders had no role in study design, data collection and analysis, decision to publish, or preparation of the manuscript.

\section{Availability of data and materials}

All the data supporting our findings is contained within the manuscript. Therefore, data sets are not shown.

\section{Authors' contributions}

Conceived and designed the experiments: SM, SG, AK, TM, and NO. Performed the experiments: MO, CK, YN, TS, MM, and AY. Analyzed data: MO, TO, and NO. Contributed reagents/materials/analysis tools: $M O, C K, Y N, T S, M M, A Y$, and YU. Wrote the paper: $\mathrm{MO}, \mathrm{TO}, \mathrm{BA}$, and NO. All authors have read and approved the final version of the manuscript.

\section{Competing interests}

The authors declare that they have no competing interests.

\section{Consent to publish}

Not applicable.

Ethics approval and consent to participate

The study was explained to all participants both verbally and in writing, and written informed consent was obtained from each participant. This study protocol was approved by the Ethics Committee of the Nagoya University Graduate School of Medicine, the Ethics Committee of Kaseki Hospital, and the Ethics Committee of Nagoya Teishin Hospital. The study was conducted in accordance with the established ethical standards of all institutions.

\section{Author details}

${ }^{1}$ Department of Psychiatry, Nagoya University Graduate School of Medicine, 65 Tsurumai-cho, Showa-ku, Nagoya, Aichi 466-8550, Japan. ² Sugiyama Jogakuen University, Nagoya, Japan. ${ }^{3}$ Graduate School of Education and Human Development, Nagoya University, Nagoya, Japan. ${ }^{4}$ Graduate School of Law, Nagoya University, Nagoya, Japan.

Received: 26 November 2015 Accepted: 15 June 2016

Published online: 07 July 2016

\section{References}

1. Robson KM, Kumar R. Delayed onset of maternal affection after childbirth. $\mathrm{Br}$ J Psychiatry. 1980;136:347-53.

2. Kumar RC. "Anybody's child": severe disorders of mother-to-infant bonding. Br J Psychiatry. 1997;171:175-81.

3. Hornstein C, Trautmann-Villalba P, Hohm E, Rave E, Wortmann-Fleischer S, Schwarz M. Maternal bond and mother-child interaction in severe postpartum psychiatric disorders: is there a link? Arch Womens Ment Health. 2006;9(5):279-84.

4. Noorlander $Y$, Bergink V, van den Berg MP. Perceived and observed motherchild interaction at time of hospitalization and release in postpartum depression and psychosis. Arch Womens Ment Health. 2008;11(1):49-56.

5. Kitamura T, Takegata M, Haruna M, Yoshida Y, Yamashita H, Murakami M, et al. The Mother-Infant Bonding Scale: Factor structure and psychological correlates of parental bonding disorders in Japan. J Child Fam Stud. 2015;24:393-401.

6. Muzik M, Bocknek EL, Broderick A, Richardson P, Rosenblum KL, Thelen $\mathrm{K}$, et al. Mother-infant bonding impairment across the first 6 months postpartum: the primacy of psychopathology in women with childhood abuse and neglect histories. Arch Womens Ment Health. 2013;16(1):29-38.

7. Cranley MS. Development of a tool for the measurement of maternal attachment during pregnancy. Nurs Res. 1981;30(5):281-4.

8. Alhusen JL, Hayat MJ, Gross D. A longitudinal study of maternal attachment and infant developmental outcomes. Arch Womens Ment Health. 2013;16(6):521-9.

9. Edhborg M, Nasreen HE, Kabir ZN. Impact of postpartum depressive and anxiety symptoms on mothers' emotional tie to their infants 2-3 months postpartum: a population-based study from rural Bangladesh. Arch Womens Ment Health. 2011;14(4):307-16.

10. van Bussel JC, Spitz B, Demyttenaere K. Three self-report questionnaires of the early mother-to-infant bond: reliability and validity of the Dutch version of the MPAS, PBQ and MIBS. Arch Womens Ment Health. 2010;13(5):373-84.

11. Brockington IF, Oats J, George S, Turner D, Vostanis P, Sullivan M, et al. A screening questionnaire for mother-infant bonding disorders. Arch Womens Ment Health. 2001;3:133-40.

12. Condon JT. The assessment of antenatal emotional attachment: development of a questionnaire instrument. Br J Med Psychol. 1993;66(Pt 2):167-83. 
13. Taylor A, Atkins R, Kumar R, Adams D, Glover V. A new Mother-to-Infant Bonding Scale: links with early maternal mood. Arch Womens Ment Health. 2005;8(1):45-51.

14. Yoshida K, Yamashita H, Conroy S, Marks M, Kumar C. A Japanese version of Mother-to-Infant Bonding Scale: factor structure, longitudinal changes and links with maternal mood during the early postnatal period in Japanese mothers. Arch Womens Ment Health. 2012;15(5):343-52.

15. Yamashita $\mathrm{H}$. The relationship between Postnatal Depression and Bonding Disorder. Arch Psychiatr Diag Clin Eval. 2014;14(1):41-8 (in Japanese).

16. Cox JL, Holden JM, Sagovsky R. Detection of postnatal depression. Development of the 10-item Edinburgh Postnatal Depression Scale. Br J Psychiatry. 1987;150:782-6.

17. Okano T, Murata M, Masuji F, Tamaki R, Nomura J, Miyaoka H, et al. Validation and reliability of Japanese version of the Edinburgh Postnatal Depression Scale (EPDS). Arch Psychiat Diag Clin Eval. 1996:7(4):525-33 (in Japanese).

18. Yamashita H, Yoshida K, Nakano H, Tashiro N. Postnatal depression in Japanese women. Detecting the early onset of postnatal depression by closely monitoring the postpartum mood. J Affect Disord. 2000;58(2):145-54.

19. Cattel R. The scree test of the number of factors. Multivariate Behav Res. 1996;1:245-76.

20. Schermelleh-Engell $\mathrm{K}$, Moosbrugger $\mathrm{H}, \mathrm{M} \quad$ ler $\mathrm{H}$. Evaluating the fit of structural equation models: Test of significance and descriptive goodnessof-fit measures. Method Psychol Online. 2003;8:23-74.

21. Kokubu M, Okano T, Sugiyama T. Postnatal depression, maternal bonding failure, and negative attitudes towards pregnancy: a longitudinal study of pregnant women in Japan. Arch Womens Ment Health. 2012;15(3):211-6.

22. Kitamura T, Takegata M, Haruna M, Yoshida Y, Yamashita H, Murakami M, et al. Depressive mood, bonding Failure, and abusive parenting among mothers with three-month-old babies in a Japanese community. J Psychiatry. 2013;3:1-7.

23. Dubber S, Reck C, Müller M, Gawlik S. Postpartum bonding: the role of perinatal depression, anxiety and maternal-fetal bonding during pregnancy. Arch Womens Ment Health. 2015;18(2):187-95.

24. Vik T, Grote V, Escribano J, Socha J, Verduci E, Fritsch M, et al. Infantile colic, prolonged crying and maternal postnatal depression. Acta Paediatr. 2009:98(8):1344-8. doi:10.1111/j.1651-2227.2009.01317.x.

25. Moehler E, Brunner R, Wiebel A, Reck C, Resch F. Maternal depressive symptoms in the postnatal period are associated with long-term impairment of mother-child bonding. Arch Womens Ment Health. 2006;9(5): 273-8. doi:10.1007/s00737-006-0149-5.

26. Sockol LE, Battle CL, Howard M, Davis T. Correlates of impaired motherinfant bonding in a partial hospital program for perinatal women. Arch Womens Ment Health. 2014;17(5):465-9. doi:10.1007/s00737-014-0419-6).

\section{Submit your next manuscript to BioMed Central and we will help you at every step:}

- We accept pre-submission inquiries

- Our selector tool helps you to find the most relevant journal

- We provide round the clock customer support

- Convenient online submission

- Thorough peer review

- Inclusion in PubMed and all major indexing services

- Maximum visibility for your research

Submit your manuscript at www.biomedcentral.com/submit

) Biomed Central 